\title{
Developments in Flow Injection-Capillary Electrophoresis Systems
}

\author{
Zhao-Lun FAng, ${ }^{*}, * \dagger$ Heng-Wu Chen, ${ }^{* *}$ Qun FAnG, ${ }^{* *}$ and Qiao-Sheng PU* \\ *Research Center for Analytical Sciences, Chemistry Department, Northeastern University, \\ Chemistry Building, Box 332, 110006 Shenyang, China \\ **Institute of Microanalytical Systems, Chemistry Department, Xixi Campus, Zhejiang University, \\ 310028 Hangzhou, China
}

\begin{abstract}
An overview is given on the recent development of combined flow injection (FI) capillary electrophoresis (CE) systems. The basic principles are treated and the advantages of the combined system, including improved throughput and reproducibility, and the potentials of incorporating various on-line separation and preconcentration systems, including filtration, dialysis, gas diffusion, and column sorption, are discussed and illustrated by applications.
\end{abstract}

(Received November 2, 1999; Accepted November 26, 1999)

Following the early contributions to capillary electrophoresis (CE) made by Mikkers et al. ${ }^{1}$ as well as Jorgensen and Lukas, ${ }^{2}$ the technique has evolved into a major analytical tool for the separation of charged compounds within the past twenty years, with related publications impressively soaring to over 6000 in late 1999. Despite the multiple and outstanding advantages of $\mathrm{CE}$ as a separation technique, including a wide range of applicable analytes, high column efficiency and resolution, low sample and electrolyte consumption, and short analysis times, some generally recognized limitations still affect its broad acceptance in routine applications. The main limitations of conventional CE may be summarized as:

(a) Discontinuous mode of sample introduction (electrokinetic, gravitational), even automated, confines the sample throughput and precision.

(b) The electrokinetic mode can "bias" sample introduction; i.e., sample components with higher electrophoretic mobilities are preferentially introduced over those with low mobilities.

(c) Low sensitivity in terms of the concentration with photometric detection owing to the short light path.

(d) Difficulties with samples containing large amounts of proteins and macromolecules when directly injected into the separation capillary, owing to fouling of the capillary walls by adsorption.

Based on our recent studies on combining the flow injection (FI) technique with $\mathrm{CE}^{3-8}$ and those conducted by Karlberg's group, ${ }^{9-14}$ these limitations can be either completely overcome or at least significantly alleviated by synchronously coupling the two techniques.

FI has proved to be a powerful and efficient means for sample introduction as well as sample pretreatment for flow analytical systems involving molecular spectrophotometry, atomic

†To whom correspondence should be addressed.

E-mail:fangzl@mail.neu.edu.cn

Q.-S. P.: on leave from Chemistry Department, Lanzhou University, Lanzhou, China.

This paper was presented at ASIANALYSIS V, Xiamen, China, May 4 - 7, 1999. spectrometry, luminometry, chromatography, and electrochemical detection. ${ }^{15}$ Nevertheless, certain technical obstacles in combining FI with CE did exist which may be summarized as:

(a) Discontinuity of sample introduction operation in conventional CE.

(b) Differences in the sample-introduction volume between the two techniques, reaching to three orders of magnitude.

(c) For safety reasons, decoupling of the applied high voltage for CE separation from the FI system is required.

In 1997, these obstacles were independently overcome by Karlberg's group ${ }^{9}$ and our group ${ }^{3}$ with a similar technical approach, i.e., the implementation of a mixed hydrodynamicelectrokinetic split-flow interface. Since then a series of on-line sample pretreatment systems based on FI principles were successfully adapted for on-line coupling to CE systems with applications to real samples. The main achievements in the field are reviewed in this article.

\section{The FI-CE Split-flow Interface and Basic FI-CE System}

The mixed hydrodynamic-electrokinetic split-flow interfaces developed by Karlberg's group ${ }^{9}$ and the most recent version developed by our group ${ }^{7}$ are shown schematically in Figs. 1a, b. Both are characterized by an electrically grounded low deadvolume split-flow cell, in which a small fraction of the sample is electrokinetically introduced into the separation capillary located in the cell when an injected sample zone in the microliter range is transported past the capillary tip by a carrier buffer using peristaltic pumps. The main differences between the two interfaces are the flow direction of the carrier in relation to the electrode and separation capillary and the mode for maintaining a constant hydrostatic level in the cell. The interface developed by Karlberg (Fig. 1b) featured a Plexiglas body with a horizontal channel for a carrier buffer and sample transport from the FI system. A separation capillary and a grounded electrode were vertically inserted into the channel. Our split-flow interface (Fig. 1a) was composed of a vertically 
positioned conical open-cell, through which the carrier buffer was introduced from the lower end and expelled by forced outflow via an upper outlet. By employing an outflow rate higher than the inflow, the liquid level was maintained constant and identical with that of the capillary outlet. Thus, hydrodynamic flow in the capillary owing to differences in the liquid levels at its two ends was kept to a minimum. The separation capillary was inserted into the cell with its tip positioned a few millimeters above the inlet, and the grounded electrode was positioned with its end above the tip of the a

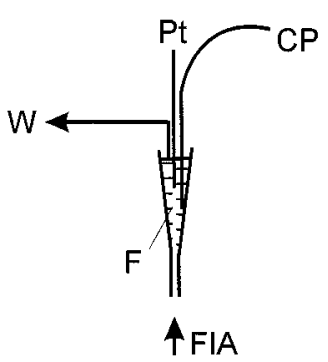

b

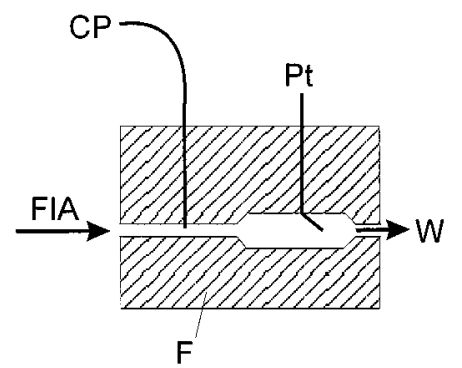

Fig. 1 Schematic diagram of split-flow interfaces for coupling FI to CE: (a) vertical design; ${ }^{3}$ (b) horizontal design. ${ }^{9}$ F, flow-cell; Pt, platinum electrode; $\mathrm{CP}$, separation capillary; W, waste. Reproduced by permission of Elsevier Science B. V. capillary so as to avoid possible interferences from gas bubbles generated at the electrode surface. An outer reservoir furnished with an outlet was used to house the conical cell and grounding electrode in the original design, ${ }^{3}$ but later $^{5}$ this was found not to be necessary. The working principle of the interface is shown in Fig. 1c.

With both interfaces, electrokinetic injection of a series of samples could be achieved in a continuous non-interrupted mode with the separation voltage applied throughout, without shifting the inlet of the separation capillary between the buffer vial and sample vial, as practiced in conventional CE.

The basic FI-CE system setup is shown schematically in Fig. 2. With benzoic acid as a model analyte, various experimental parameters that might affect the performance of the coupled FICE system were examined by Fang et al., ${ }^{3}$ who found that the sample volume and carrier flow-rate of the FI system had significant effects on both the separation efficiency and the sensitivity of CE. Within the range of the frequently used FI parameters, the separation efficiency increased and the sensitivity, expressed in peak height, decreased along with a reduction in the sample volume and increase in the carrier flowrate. This is because both the sample volume and the flow-rate determines the residence time of the sample plug in the splitflow interface, and thus the amount of the sample electrokinetically introduced into the capillary. Basically similar observations were made by Kuban et al. ${ }^{9}$ Both groups found that the precision of CE determinations was significantly improved due to avoiding interruption of the separation voltage,

A
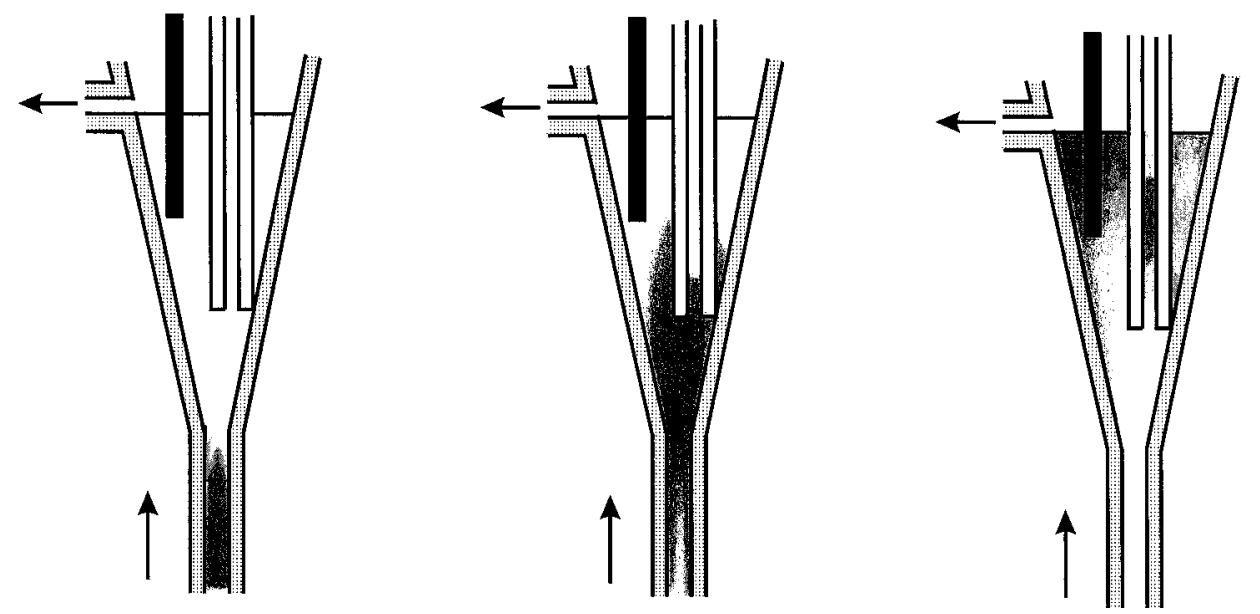

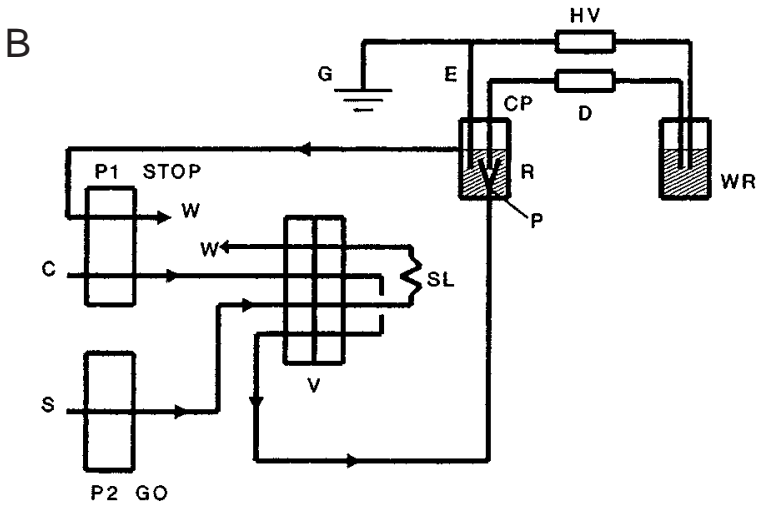

(a) LOAD

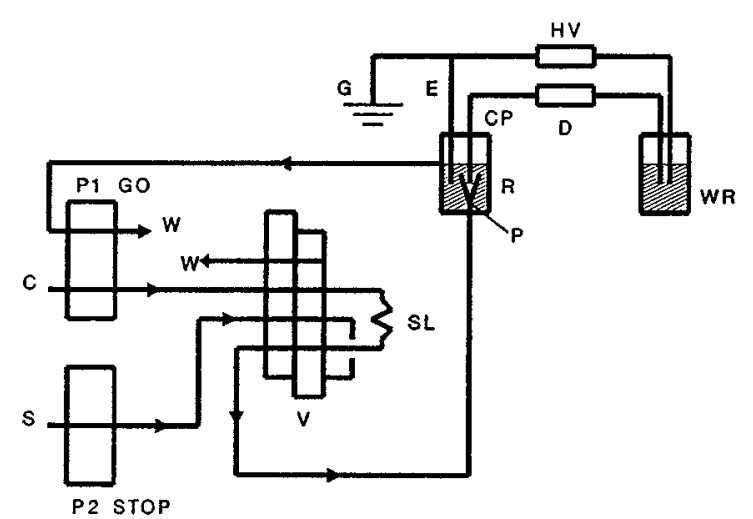

(b) INJECTION

Fig. 2 a: Working principles of the vertical FI-CE interface. b: basic manifold configuration of FI-CE system. ${ }^{3}$ Reproduced by permission of Elsevier Science B. V. 

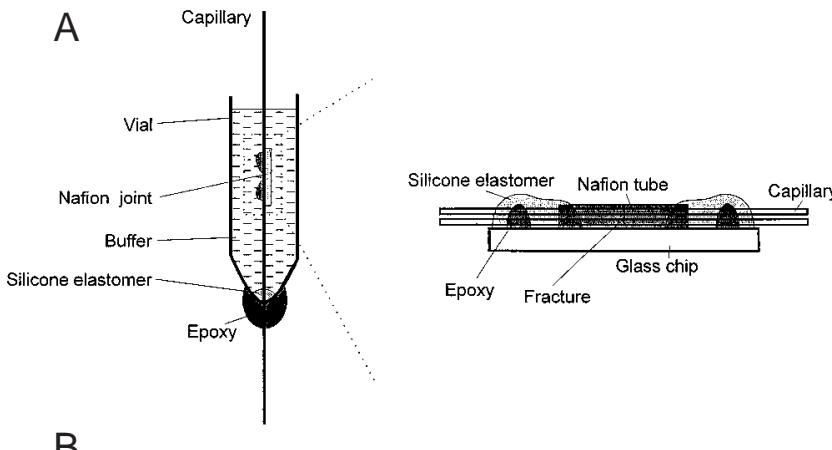

a)

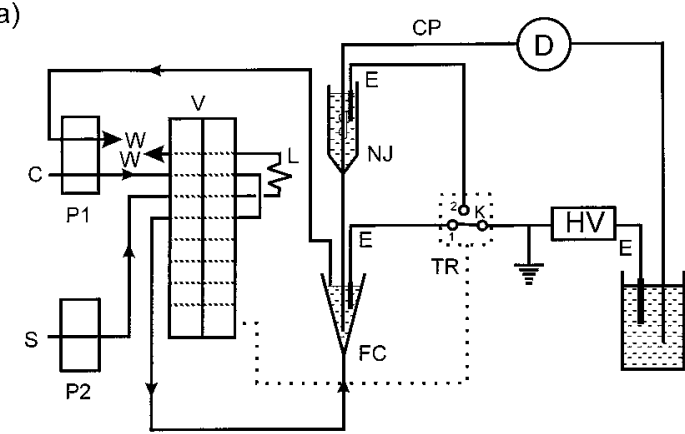

b)

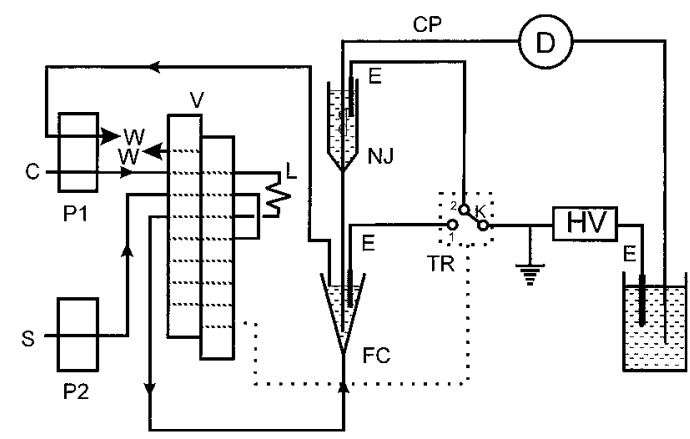

Fig. 3 (A) Structure of the Nafion joint. (B) The FI-CE system with EOF traction injection. (a) Fill position; (b) injection position. $\mathrm{P} 1, \mathrm{P} 2$, peristaltic pumps; $\mathrm{V}$, injection valve; L, sample loop; C, carrier (buffer), S, sample; W, waste; CP, separation capillary; E, platinum electrode; K, microswitch; FC, flowcell; NJ, Nafion joint; HV, high voltage supply; D, detector; R, buffer reservoir; TR, time relay. ${ }^{8}$ Reproduced by permission of Elsevier Science B. V.

and that overlapping of electropherograms of multiple samples was feasible in order to increase the sample throughput. The performance of the basic FI-CE system was illustrated by our group by applying the system to the separation and determination of magnolol and honokiol in Chinese herbal fomulations, ${ }^{17}$ and by Karlberg's group to the determination of inorganic anions in water samples by indirect UV detection. ${ }^{9}$

The advantages of the FI-CE system over the conventional methods may be summarized as:

(a) Outstanding reproducibility in retention time, peak area and peak height (RSDs of better than 2.5\% for the peak height were reported by both Karlberg's group ${ }^{9}$ and our group ${ }^{3}$ ).

(b) Enhanced sample throughput through a simplification in operation, usually by at least a factor of two, when separations are effected within a few minutes.

(c) To further increase the sampling frequency, more than one electropherogram may be overlapped, provided peaks of interest do not overlap, i.e. one or two more samples may be injected before the last peak of the first sample has been detected.

(d) Large potentials in coupling FI on-line separation and preconcentration techniques to $\mathrm{CE}$ to enhance the selectivity and sensitivity.

The main shortcoming of the FI-CE split-flow interface lies in the relatively large sample consumption of a few decade microliters, which though not excessive, is still significantly more than the minimum volume required for conventional $\mathrm{CE}$. A further drawback in the interface designs shown in Fig. 1 is the limitations associated with bias effects inherent in electrokinetic injection.

To overcome the bias effect of the split-flow interface based on electrokinetic injection, Karlberg's group ${ }^{13}$ developed an interface based on hydrodynamic injection, in which the carrier flowing out of the interface was controlled by a pinch valve. When the sample plug in the carrier passed the capillary inlet in the FI-CE interface, with high voltage supply disconnected, the pinch valve was shut off for a defined period of time (usually $0.1-1 \mathrm{~s}$ ), during which a fraction of the sample was forced into the capillary under the developed pressure. High voltage was resumed across the separation capillary after the injection, and separation was initiated. With two programmable time relays, one controlling the pinch valve and the other the voltage supply, synchronously with an injection valve, repetitive sample injections during the course of one electrophoretic run was achieved. However, this created hydrodynamic flows within the capillary which deteriorated the separation efficiency to some extent.

More recently, we developed a bias-free split-flow sampleintroduction interface for FI-CE based on introducing the sample into the capillary by electroosmotic flow traction instead of electrophoretic migration of analytes. ${ }^{8}$ A Nafion joint, which only allowed ions to penetrate, was implemented at a capillary fracture about $2 \mathrm{~cm}$ downstream of the capillary inlet. The joint was immersed in a buffer solution contained in a plastic vial (Figs. 3a, b), in which a platinum electrode was inserted. The capillary inlet was threaded through the bottom of the vial, and inserted into the conical flow-cell through which the carrier and injected sample were transported. The complete FI-CE system is shown schematically in Fig. 3. When a sample plug reached the flow-cell, the electrode in the Nafion joint vial was automatically connected to the high-voltage supply, and the electroosmotic flow generated by the voltage applied across the capillary fracture and its outlet drew into its inlet a fraction of the sample plug flowing pass the inlet. Since electrical fields were almost absent in the split-flow interface, bias effects owing to electromigration of the analytes were avoided. After the sample zone had passed the interface, the high-voltage supply was applied across the inlet and outlet of the capillary and electrophoretic separation was initiated. With this arrangement the peak-area ratios of two model compounds, dimethyl sulfoxide and benzoic acid, obtained using the electroosmotic traction system were identical to those obtained with gravity injection. The FI-CE system with a bias-free split-flow interface was applied to the separation and determination of caffeine, theobromine and theophylline in various beverage samples with satisfactory recoveries in the $92-107 \%$ range.

\section{Micro-chip Based FI-CE System}

Recently, we demonstrated the advantages of combining FI with $\mathrm{CE}$ in a micro-chip based CE system. ${ }^{16}$ A micro-volume sequential injection (SI) sample-introduction system was 

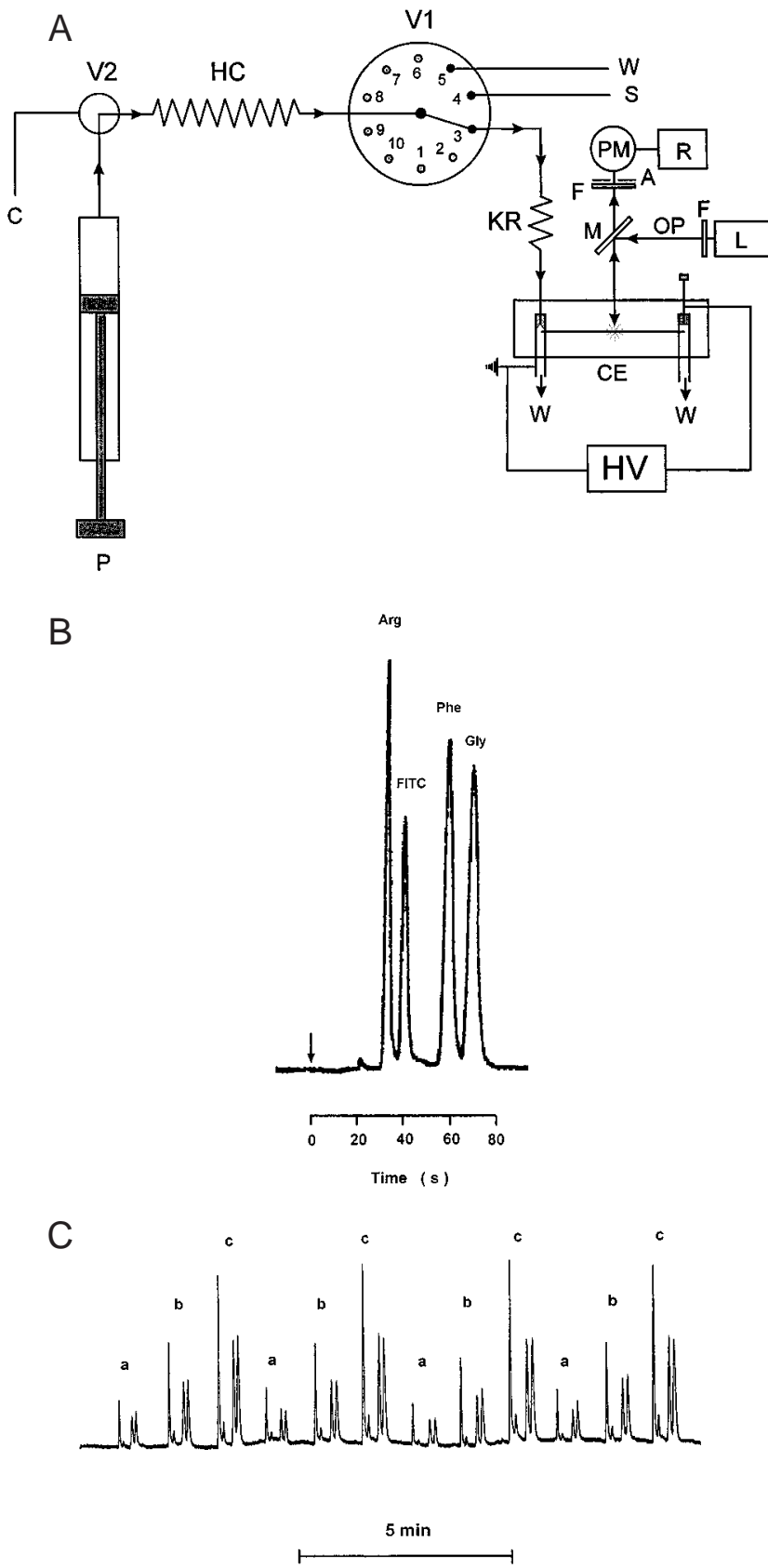

Fig. 4 (A) Schematic diagram of the SI-sample introduction microfluidic CE system. P, syringe pump; V1, multi-position selector valve; V2, two-way valve; HC, holding coil; C, carrier (buffered working electrolyte); S, sample; W, wastes; KR knotted reactor; $\mathrm{CE}$, microfluidic $\mathrm{CE}$ system; $\mathrm{HV}$, high voltage power supply; L, laser source; M, dichroic mirror; OP, optical fiber; F, filters; A, pinhole aperture; PM, photomultiplier; R, chart recorder. (B) Typical electropherograms of a mixture of three FITC-labeled amino acids (100 $\mu \mathrm{M}$ each) obtained at separation length of $25 \mathrm{~mm}$, and electric field strength of $250 \mathrm{~V} \mathrm{~cm}^{-1}$. Carrier (buffer) solution, $4 \mathrm{mM}$ sodium tetraborate ( $\mathrm{pH}$ 9.2), flow-rate, $2 \mathrm{ml} \mathrm{min}^{-1} ; 3.3 \mu \mathrm{l}$ sample. (C) FITC labeled amino acid mixture recorded with four cycles of sequentially injected 25, 50, and $100 \mu \mathrm{M}$ samples. ${ }^{16}$ Reproduced by permission of Elsevier Science B. V.

employed in order to further minimize the injected sample volume. An H-configuration arrangement was adopted in this case to accommodate the split-flow interface and short separation capillary on a single $20 \times 70 \times 1 \mathrm{~mm}$ glass chip (Fig. 4A). With a short separation capillary of $60 \mathrm{~mm}$ (effective separation length, $25 \mathrm{~mm}$ ) the continuous delivery of microliter samples by a suitable carrier was shown to be feasible at throughputs of $40-60 \mathrm{~h}^{-1}$ with less than $3 \%$ carryover between neighboring samples. The system was studied using a mixture of amino acids labeled with fluorescein isothiocyanate (FITC) and laser-induced fluorescence detection; sample volumes as low as $1.67 \mu \mathrm{l}$ were injected into the split-flow interface. Chart recordings obtained during the separation of fluorescein isothiocyanate(FITC)-labeled amino acids, arginine, phenylalanine, and glycine are shown in Figs. 4B, C. The injected sample volume was $3.3 \mu \mathrm{l}$, and the separation time was $80 \mathrm{~s}$ at $250 \mathrm{~V} \mathrm{~cm}^{-1}$ field strength, yielding a sampling frequency of 48 $\mathrm{h}^{-1}$, and a precision of $2.9 \%$ RSD for arginine, with baseline separation of the three amino acids and the fluorescence dye.

\section{FI-CE Systems Involving On-line Separation and Preconcentration}

\section{General}

As stated in the previous section, one of the important advantages of the combined FI-CE system is the possibility of readily employing well-documented on-line FI sample pretreatment techniques, mainly involving on-line separations and preconcentrations, ${ }^{15}$ to enhance the performance of $\mathrm{CE}$ procedures. The systems thus-far reported are schematically presented in Fig. 5, including on-line sample filtration, dialysis, gas diffusion, and column sorption. A common feature of such systems is that the sample pretreatment may be operated independently, and in parallel with the CE separation; sample wastes resulting from the pretreatment are not introduced into the separation capillary. The FI separation and CE systems may also be optimized separately, except that the sample matrix (solvent, buffer and $\mathrm{pH}$ ) following separation should conform with the requirements of the $\mathrm{CE}$ separations. The sample frequencies are usually not significantly degraded by the incorporation of an on-line separation, and are often even higher than in the $\mathrm{CE}$ procedures using conventional sample introduction techniques.

\section{FI-CE system with on-line filtration}

Capillaries used in $\mathrm{CE}$, usually with inner diameters of less than $100 \mu \mathrm{m}$, are easily fouled by solid particles suspended in sample solutions. When $\mathrm{CE}$ is used to analyze liquid samples with suspended fine solid particles, isolation of these particles from the solution by filtration is required. Manually operated batchwise filtration procedures are often tedious and susceptible to contamination from the apparatus and the environment. When a dynamic process, for instance drug dissolution, is to be monitored, batchwise sample filtration cannot meet the requirements of real-time analysis. On-line filtration can be readily incorporated in the combined FI-CE system. Chen and Fang $^{6}$ developed an on-line filtration-FI-CE for monitoring a drug dissolution process, by incorporating a $75 \mathrm{~mm}^{2}$ membrane filter at the tip of the sampling probe of the injection valve, as depicted in Figs. 5 and 6; a fully automated dissolution testing system was produced for multicomponent monitoring, capable of a high-resolution dissolution process (see Applications section for details). Non-dissoluted excipients in the tested drug preparations were separated during loading of the sample loop, the surplus of filtered sample being directed back to the dissolution vessel to reduce sample consumption. Thus, only a $25 \mu \mathrm{l}$ sample was consumed for each measurement. 


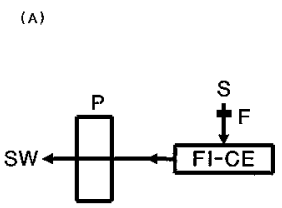

(c)

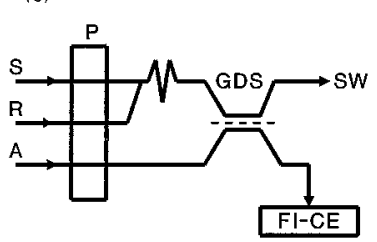

(D)

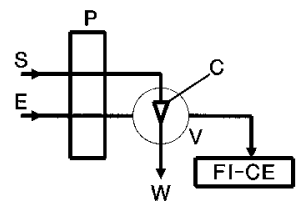

(E)

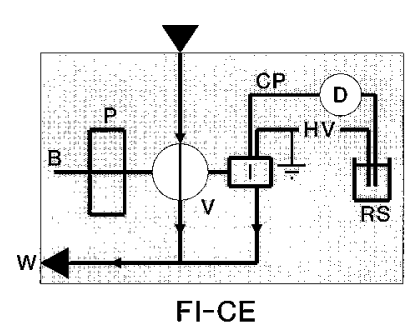

Fig. 5 Schematic diagram of various on-line separation systems connected to FI-CE. (A), on-line filtration mode; ${ }^{17}$ (B) on-line dialysis mode; ${ }^{10}(\mathrm{C})$ on-line gas diffusion mode; ${ }^{11}$ (D) on-line column sorption mode; ${ }^{5,14}$ (E) FI-CE basic system. P, pump, S, sample; SW, sample waste; FI-CE, combined FI-CE system; F, membrane filter; DS, on-line dialysis membrane separator; A, acceptor stream; R, reagent for generation of gas phase; GDS; on-line gas diffusion membrane separator; $\mathrm{C}$, sorption column; E, eluent; $\mathrm{V}$, injector valve; W, waste; B, buffer/carrier; I, interfacing flow-cell (see Fig. 1); CP, separation capillary; HV, high voltage supply; RS, outflow electrolyte reservoir; D, detector. Reproduced by permission of Elsevier Science B. V.

\section{FI-CE system with on-line dialysis}

The inner surface of the capillary tends to adsorb biomacromolecules in biological samples, leading to changes in the surface properties, and hence the electroosmotic flow. Interfering macromolecular constituents may be separated by dialysis, which, similar to filtration discussed in the previous section, has certain limitations when operated manually. Kuban and Karlberg ${ }^{10}$ successfully coupled on-line dialysis to FI-CE for the analysis of small anions (see Fig. 5b). Samples were continuously pumped into the donor channel of a dialyzer incorporating a dialysis membrane sandwiched between an acceptor stream channel and a donor stream channel. The outflowing acceptor stream containing the separated analytes was loaded into the sample loop and injected into the FI-CE system. Multiple injections in one electrophoretic run increased the sample throughput rate. The repeatability of the on-line dialysis FI-CE system for chloride, nitrate and sulfate, $100 \mathrm{mg}$ $1^{-1}$ each in a standard solution, was within $3.3 \%$ RSD $(n=7)$ using a peak height evaluation. The effectiveness of the separation was tested by determining small anions in such samples as milk and fruit juice without any pretreatment before sample injection.

\section{FI-CE systems with on-line gas-diffusion}

On-line gas diffusion has also been coupled by Kuban and Karlberg, ${ }^{11}$ to a FI-CE system for separating volatile analytes from complicated sample matrices (see Fig. 5c). In contrast to
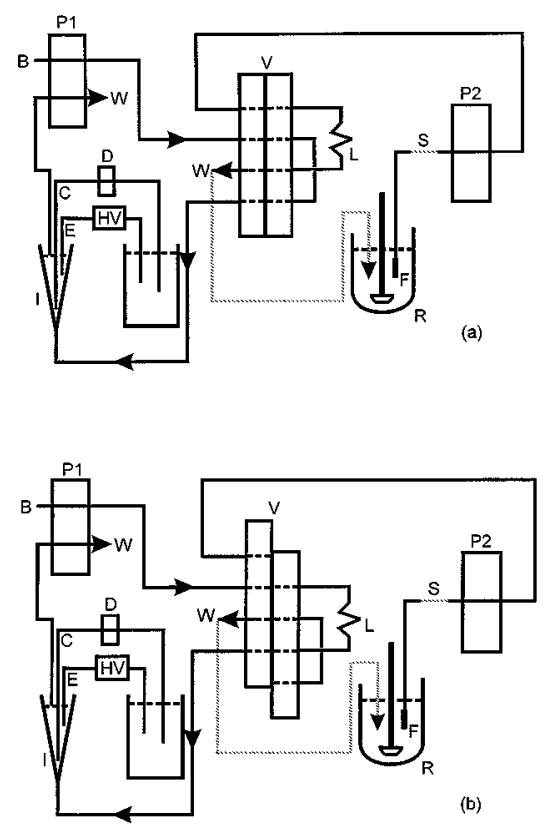

Fig. 6 Schematic diagram of the on-line filtration FI-CE system for dissolution testing (a) sampling; (b) injection. B, buffer solution; C, separation capillary; D, UV detector; E, anode connected to high voltage supply; F, filter, HV, high voltage supply; I, split-flow interface cell; L, sampling loop; P1 and P2, peristaltic pumps; V, injection valve; S, sampling probe; $\mathrm{W}$ and $\mathrm{W}^{\prime}$, wastes; $\mathrm{R}$, dissolution vessel. ${ }^{6}$ Reproduced by permission of Elsevier Science B. V.

dialysis, where analytes are isolated from the matrix according to their molecular size, the gas-diffusion process usually requires conversion of ionic analytes into their respective gaseous species, which can penetrate a semipermeable membrane. The separated analyte species are then trapped in the acceptor phase by conversion of the gaseous species into ions. Thus, samples are on-line merged with a strong acid in a flow system to transform, e.g., sulfite and carbonate into sulfur dioxide and carbon dioxide. The transformed gaseous analytes permeate through a microporous PTFE membrane into an acceptor stream of a tris buffer solution. The continuous flowing acceptor phase was led into the sample loop of the FI$\mathrm{CE}$ system and injected into the split-flow interface. Multiple sample injections were performed in one uninterrupted electrophoretic run, resulting in a typical sampling frequency of $15 \mathrm{~h}^{-1}$, with RSD's in the range of $1.8-3.8 \%$. A large range of beverages were analyzed for volatile species under acidic conditions without further sample pretreatment.

FI-CE system with on-line solid phase extraction preconcentration Despite its merits, such as high separation power and speed of analysis, low sample consumption and operational cost, one of the shortcomings of CE lies in its low concentration sensitivity. When UV detection is employed, the detection limit in the range of $10^{-5}-10^{-6} \mathrm{~mol} \mathrm{l}^{-1}$ is far above the levels required for trace analysis. On-capillary stacking and field-amplified sample injection can concentrate analytes by $2-3$ orders of magnitude, but usually require critical experimental conditions. Samples should have a uniform matrix with low conductivity, which may be difficult to achieve. Thus, for the determination of trace constituents in biomedical and environmental analysis, analyte enrichment and sample cleanup are inevitable before attempting $\mathrm{CE}$ analysis. Among the FI-CE on-line separation systems described in the previous sections, only gas-diffusion may be 


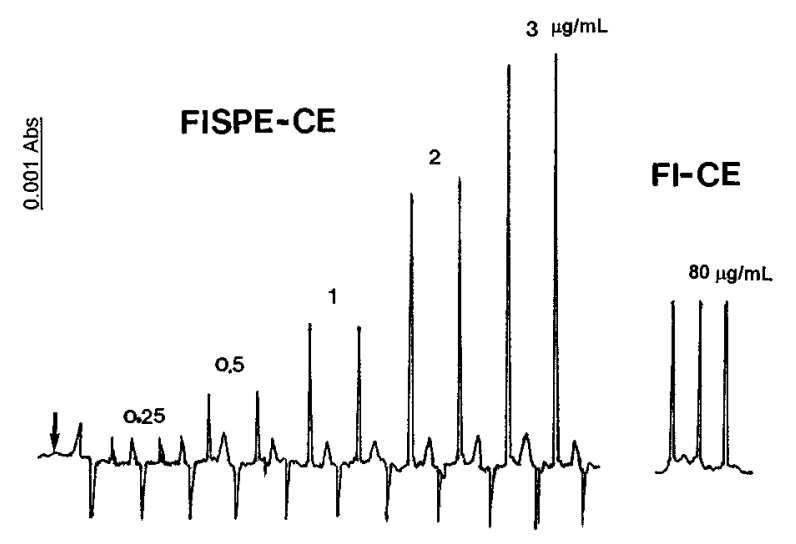

\section{$20 \mathrm{~min}$}

Fig. 7 Duplicate recorder traces of (a) standard solutions of 0.25 , $0.50,1.00,2.00,3.00 \mu \mathrm{g} \mathrm{ml}^{-1}$ pseudoephedrine for FI-CE with SPE preconcentration; arrow points to peak position of reagent blank; (b) a standard solution of $80 \mu \mathrm{g} \mathrm{ml}^{-1}$ pseudoephedrine obtained by FI-CE without preconcentration. Conditions for on-line preconcentration

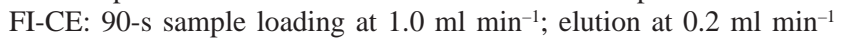
with $40 \% 62.5 \mathrm{mM}$ acetate buffer $(\mathrm{pH} 4.0)$ and $60 \%$ acetonitrile; eluate volume $25 \mu \mathrm{l}$; preceding water zone $25 \mu \mathrm{l}$; phosphate buffer carrier (pH 6.8), $0.5 \mathrm{ml} \mathrm{min}{ }^{-1}$ flow-rate; $12 \mathrm{kV} ; 28-\mathrm{cm}, 75 \mu \mathrm{m}$ i.d. silica capillary; $210 \mathrm{~nm}$ wavelength. ${ }^{5}$ Reproduced by permission of Elsevier Science B. V.

used for preconcentration purposes. ${ }^{15}$ Yet, the latter is limited only to constituents which can be readily transformed into a gaseous species. FI on-line ion-exchange and solid phase extraction (SPE) using mini-columns have proved to be efficient means for preconcentration purposes applicable to a wide range of analytes. ${ }^{15}$ We succeeded in developing an on-line column preconcentration system for FI-CE based on sorption extraction using a micro-column packed with $\mathrm{C}_{18}$, which was incorporated in a multi-channel injector valve (Fig. 5d). During sample loading, the micro-column was switched out of connection with the split-flow interface. The analyte was sorbed on the column while the sample effluent was directed to waste, so that no sample matrix was allowed to reach the CE system. During elution, the valve was switched to connect the micro-column to a $40 \mu \mathrm{l}$ loop in the valve (not shown in Fig. 5D). With appropriate timing, a heart-cut of the effluent zone with the highest analyte concentration was accomplished and stored in the loop. The concentrate plug was then delivered by a carrier flow into the split-flow interface. When using a model analyte pseudoephedrine to study the performance of the system, the composition of the eluent significantly influenced the sensitivity of the system. This was due to the different strengths of the eluents as well as to differences in their conductivity, which might cause further on-capillary concentration resulting from stacking effects and field amplified sample introduction. Thus, in the FI-CE on-line column preconcentration system, sensitivity enhancements may be achieved both by increasing the phase transfer efficiency and by electrophoretic effects induced by modifying the sample matrix during elution. Enhancement factors of 66 and 182 were achieved for pseudoephedrine with sample loading times of 1.5 and $4 \mathrm{~min}$, respectively, at a loading rate of $1 \mathrm{ml} \mathrm{min}^{-1}$ (Fig. 7). A detection limit of $0.03 \mu \mathrm{g} \mathrm{l}^{-1}$ (3 $\sigma$ was obtained for $1.5 \mathrm{~min}$ loading, which yielded a high sampling frequency of $10 \mathrm{~h}^{-1}$, including the preconcentration and CE separation.

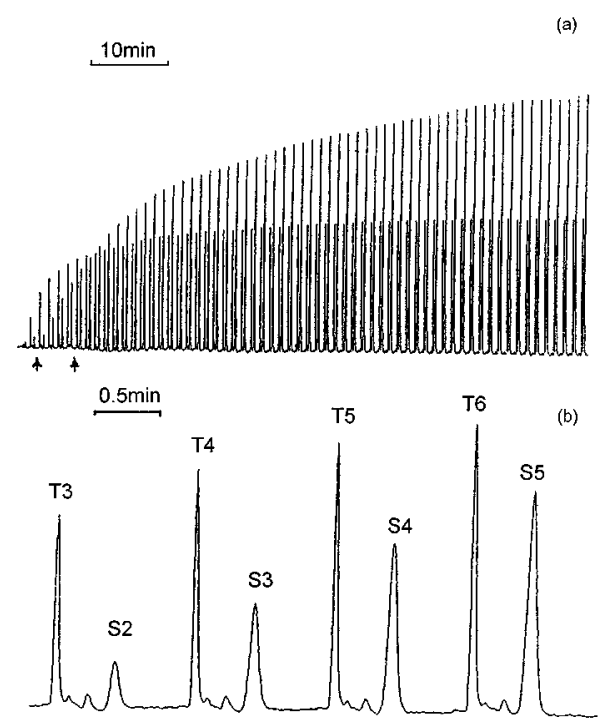

Fig. 8 Electropherograms of drug-dissolution testing with FI-CE employing on-line membrane filtration. ${ }^{6}$ (a) Complete dissolution run; (b) expanded view of time range between arrows in (a); T3, T4, T5, T6 are the TMP peaks for the 3rd, 4th, 5th and 6th injections respectively, and S2, S3, S4, S5 are the SMZ peaks for the 2nd, 3rd, 4th and 5th injections, respectively. FI conditions: sample volume, $25 \mu \mathrm{l}$; carrier (running buffer) flow rate, $1.2 \mathrm{ml} \mathrm{min}^{-1}$; CE conditions: $75 \mu \mathrm{m}$ i.d. uncoated silica capillary; $29 \mathrm{~cm}$ length $(14.5 \mathrm{~cm}$ effective); separation voltage, $10 \mathrm{kV}$; running buffer, $75 \mathrm{mmol} \mathrm{l}^{-1}$ phosphate ( $\mathrm{pH}$ 6.5). Reproduced by permission of Elsevier Science B. V.

More recently, Arce et al. ${ }^{14}$ used a similar FI-CE system for the on-line ion-exchange preconcentration of nitrite, nitrate, bromide and iodide with UV absorbance detection. Five-fold enrichments were achieved with a precision of about 5\% RSD.

\section{Some Applications}

\section{Chiral separations}

The performance of the FI-CE combined system was tested by Liu and Fang ${ }^{4}$ by applying it to the chiral separation of intermediate enantiomers in chloramphenicol synthesis. $\beta$ Cyclodextrin and derivatives, used as chiral selectors, were added to the separation buffer, which also functioned as the FI carrier. Baseline resolution of enantiomers of the rac-threo bases 2-amino-1-( $p$-nitrophenyl-1,3-propanediol) was achieved with a theoretical plate number of $1.2 \times 10^{6}$, and a sample throughput of $15 \mathrm{~h}^{-1}$, which was higher than conventional CE by a factor of five. The peak-height reproducibility was $2-3 \%$ RSD, which was also significantly better than that obtained by conventional CE (7 - 10\% RSD).

\section{Continuous monitoring of multicomponents in drug dissolution process}

The high sampling frequencies of FI-CE systems make it possible to use $\mathrm{CE}$ in process analysis for the simultaneous monitoring of multianalytes. The on-line separation capabilities are extremely useful in dealing with process samples with complicated matrices. Such potentials were demonstrated by applying the on-line filtration FI-CE system described previously regarding drug dissolution studies. ${ }^{5}$ Sulfatrim tablets containing trimethoprim (TMP) and sulfamethoxazole (SMZ) were used for the study. Dissolution was carried out in $0.1 \mathrm{~mol}$ 


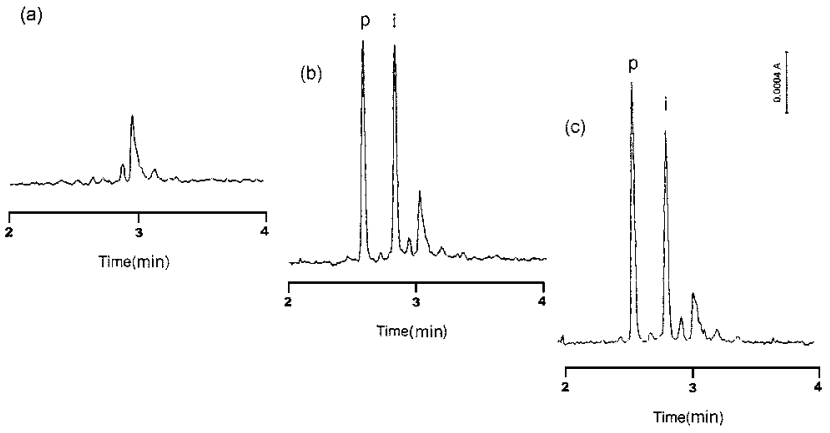

Fig. 9 Electropherographs of the plasma samples of a volunteer. (a) before oral administration; (b) blank spiked with $0.25 \mu \mathrm{g} \mathrm{ml}^{-1}$ pseudoephedrine and $0.125 \mu \mathrm{g} \mathrm{ml}^{-1}$ cimetidine; and (c) $1.5 \mathrm{~h}$ after oral administration of $60 \mathrm{mg}$ pseudoephedrine. FI-SPE-CZE conditions as in the text. $200 \mathrm{mmol} \mathrm{1}^{-1}$ acetate buffer $(\mathrm{pH} 4.4)$ containing 0.1\% PEG-6000 used as carrier and running buffer; $p$ indicates pseudoephedrine peaks, and $\mathrm{i}$, cimetidine peaks. ${ }^{7}$ Reproduced by permission of Elsevier Science B. V.

$\mathrm{1}^{-1} \mathrm{HCl}$ medium, and $\mathrm{CE}$ separation was performed in a short capillary of $14.5 \mathrm{~cm}$ effective length at a constant voltage of 10 $\mathrm{kV}$. In order to improve the resolution of the dissolution process, the sampling frequency was increased by a factor of two through partially overlapping the separation zones of neighboring samples. A high sample throughput of $60 \mathrm{~h}^{-1}$ was achieved for single-vessel dissolution. The precision achieved for 72 determinations of constant concentration dissolution solution were $1.6 \%$ and $0.8 \%$ RSD with a peak-height evaluation for TMP and SMZ, respectively. Typical chart recordings of the dissolution process using the on-line filtration-FI-CE system are shown in Fig. 8, revealing the differences in the dissolution properties of the two analytes, particularly in the earlier dissolution phase. The detailed information on the drug dissolution process obtained using the FI-CE system may be useful for the development of new drug formulations and for the quality control of pharmaceuticals.

Determination of pseudoephedrine in plasma samples with online separation and preconcentration

The potentials of FI-CE in providing sensitive and rapid methods for the analysis of biosamples with complicated matrices were recently demonstrated by a determination of pseudoephedrine in human plasma. In order to make such determinations using plasma samples obtained from volunteers orally administrated with the drug, further improvements had to be made in the on-line solid-phase extraction FI-CZE system, described previously. ${ }^{7}$ Firstly, an intermediate water-washing step was added following sample loading so that inorganic ions from salt remaining in the micro-column after loading of deproteinized plasma could be flushed out before elution, so as to ensure uniform conductivity and conditions for electrostacking for the eluate sample. Secondly, the enrichment factor was further enhanced by increasing the loaded sample volume and by increasing the electrostacking effects through an increase in the carrier/separation buffer concentration. Thus, an enhancement factor of 286 was achieved in comparison to FICZE without column preconcentration and on-capillary stacking, and a detection limit of $12 \mathrm{~g} \mathrm{l}^{-1}$ pseudoephedrine for plasma samples was obtained at a sample throughput of $10 \mathrm{~h}^{-1}$. Thirdly, $0.1 \%$ PEG- 6000 was added to the buffer solution to reduce capillary contamination caused by the adsorption of macromolecular components during repetitive injection of plasma samples. Using cimetidine as an internal standard, a relative standard deviation of $1.2 \%(n=10)$ at a concentration level of $0.5 \mu \mathrm{g} \mathrm{ml}^{-1}$ pseudoephedrine was obtained, and interday and intra-day recoveries were in the range of $95-98 \%$ and $92-101 \%$, respectively. The improved system was successfully applied to a pharmacokinetic study of pseudoephedrine in the plasma of healthy volunteers orally administrated with a normal dose of the drug (Fig. 9).

\section{Future Prospects}

Owing to outstanding improvements in the performance of $\mathrm{CE}$ systems both in terms of robustness in operation, precision, the sample throughput and sensitivity (via on-line preconcentration and exploiting electrostacking effects) of FI sample introduction may in the near future become the standard mode of sample introduction for CE. Miniaturization and integration of the combined system on microfabricated-chips may further enhance the performance, and form the basis for the development of portable CE systems that are readily applicable to such tasks as in vivo continuous monitoring of bio-active analytes in body fluids and industrial process control

\section{References}

1. F. E. P. Mikkers, F. M. Everaerts, and T. P. E. M. Verheggen, J. Chromatogr., 1979, 169, 11.

2. J. W. Jorgensen and K. D. Lukacs, Anal. Chem., 1981, 53, 1298.

3. Z.-L. Fang, Z. -S. Liu, and Q. Shen, Anal. Chim. Acta, 1997, 346, 135

4. Z.-S. Liu and Z. -L. Fang, Anal. Chim. Acta, 1997, 353, 199.

5. H.-W. Chen and Z. -L. Fang, Anal. Chim. Acta, 1997, 355, 135 .

6. H.-W. Chen and Z. -L. Fang, Anal. Chim. Acta, 1998, 376, 209.

7. H.-W. Chen and Z. -L. Fang, Anal. Chim. Acta, 1999, 394, 13.

8. Q.-S. Pu and Z.-L. Fang, Anal. Chim. Acta, 1999, 398, 65.

9. P. Kuban, A. Engstrom, J. C. Olsson, G. Thorsen, R. Tryzell, and B. Karlberg, Anal. Chim. Acta, 1997, 337, 117.

10. P. Kuban and B. Karlberg, Anal. Chem., 1997, 69, 1169.

11. P. Kuban and B. Karlberg, Talanta, 1998, 45, 477.

12. P. Kuban, K Tennberg, R. Tryzell, and B. Karlberg, J. Chromatogr., 1998, 808, 219

13. P. Kuban, R. Pirmohammadi, and B. Karlberg, Anal. Chim. Acta, 1999, 378, 55.

14. L. Arce, P. Kuban, A. Rios, M. Valcarcel, and B. Karlberg, Anal. Chim. Acta, 1999, 390, 39.

15. Z.-L. Fang, "Flow Injection Separation and Preconcentration", 1999, VCH Publishers, Weinheim.

16. Q. Fang, F.-R. Wang, S.-L. Wang, S.-S. Liu, S.-K. Xu, and Z.-L. Fang, Anal. Chim. Acta, 1999, 390, 27.

17. Q. Shen and Z.-L. Fang, Fenxi Shiyanshi, 1998, 17(2), 1. 